\title{
Prediction Modeling and Sensitivity Analysis of Robot Bone Milling Temperature Operated by a Doctor
}

\author{
Heqiang Tian $\mathbb{I D}^{1},{ }^{1}$ Jingbo Pan, ${ }^{1}$ Yu Gao, ${ }^{1}$ Xiaoqing Dang $\mathbb{D}^{\circ},{ }^{2}$ Bin Tian, \\ Debao Meng, ${ }^{1}$ and Yanan Yao ${ }^{1}$ \\ ${ }^{1}$ Shandong University of Science and Technology, Qingdao 266590, China \\ ${ }^{2}$ Qingdao Municipal Hospital Group, Qingdao 266073, China \\ Correspondence should be addressed to Xiaoqing Dang; skd994339@sdust.edu.cn
}

Received 28 June 2021; Revised 9 September 2021; Accepted 27 September 2021; Published 14 October 2021

Academic Editor: Xiaoliang Jin

Copyright (C) 2021 Heqiang Tian et al. This is an open access article distributed under the Creative Commons Attribution License, which permits unrestricted use, distribution, and reproduction in any medium, provided the original work is properly cited.

Bone milling is a common method in robot orthopedic surgery. However, excessive milling temperature will cause thermal necrosis of bone cells and tissues. It is necessary to carry out further research and analysis on the robot bone milling process considering the lamina milling skills of spinal surgeons and clinical practice to reduce the damage to bone cells and nearby tissues and obtain good cutting surface quality. Considering the randomness of milling parameters during operation, a prediction method of milling temperature model for ball milling cutter considering the doctor's surgical skills was proposed based on response surface method. Because of material anisotropy and microstructure difference between the cortical bone and cancellous bone, this paper would analyze the influencing factors in different bone layers to establish the prediction model of milling temperature in the segments of cortical bone and cancellous bone. Also, the influence and distribution of milling parameters on temperature in three cutting modes such as parallel cutting mode, cross cutting mode, and vertical cutting mode in the cortical bone region were analyzed. The parameter sensitivity of the milling temperature prediction model was analyzed by the Sobol method, and the influence of the input parameters on the output milling temperature was analyzed quantitatively.

\section{Introduction}

In recent years, medical robot has become the research hotspot of medicine and robot. Because the bone has the characteristics of rigid structure and not easy to deform and the bone image obtained before surgery has high coincidence rate and repeatability with the actual anatomical structure during surgery, bone surgery has become the main application field of robot technology [1]. Robot orthopedic surgery commonly uses ball milling cutter for bone removal, because ball milling with sharp edge, high strength, and impact resistance can disperse the cutting force and control the cutting depth with higher cutting efficiency and better cutting quality [2]. Bone tissue is an anisotropic and heterogeneous biomass composite material composed of bioactive bone cells. In the process of bone milling, excessive milling temperature will cause thermal necrosis of bone cells and tissues [3]. The surface quality of bone milling directly affects bone regeneration and implant fixation and also plays a crucial role in the surgery success [4].

It is the key to avoid excessive temperature in the process of bone cutting in modern surgery. Through theoretical analysis of the mechanism of bone cutting, the mathematical prediction model of cutting temperature can be established, and the optimal cutting parameters can be found by optimizing the prediction model, which can minimize the bone tissue damage in the process of bone processing. The bone cutting temperature model can also be used for bone processing state recognition and optimization of cutting parameters setting of each layer of bone. The related work has been paid more attention by scholars all over the world. Federspil et al. obtained optimized parameters for drilling the calvarian or mastoid bone procedure and established the first setup for robotic milling of the lateral skull base by conducting experimental milling of the skull base on two human specimens using a hexapod robot [5]. Zhang et al. deduced the thermal generation rate and other parameters 
in reverse by a thermal model and then used the finite element model to simulate the temperature distribution during the skull grinding, which established the basis for obtaining the optimal parameters [6]. Al-Abdullah et al. developed an artificial neural network force model to estimate the milling force of different bone densities as a function of the milling feed rate and spindle speed based on a series of bone milling experiments with commercial artificial bones [7]. Kianmajd et al. used CAM to calculate the position of the milling cutter and the material removal rate to estimate the robot milling force along the entire path of the cutter [8]. Feldmann et al. studied the basic principle of orthogonal cutting processing of cortical bone and cutting force, temperature rise, and chip formation under two different anterior angles and six different cutting depths to establish a mechanical model [3]. Shin and Yoon established a prediction model to estimate the maximum grinding temperature and heat distribution by conducting the grinding on the fresh bovine femur [9]. Sugita et al. pointed out that the cutting temperature increased with the increase of feed speed, estimated the heat distribution inside the cortical bone, regarded the grinding head as a linear heat source moving on a semi-infinite plane (bone surface), and theoretically calculated the cutting temperature distribution [10]. Bruce et al. predicted the bone milling temperature in neurosurgery based on the thermal energy conversion equation and the finite element heat transfer model of the linear correlation between the bone milling heat and the electric energy input of the motor [11]. Al-Abdullah et al. studied the robot milling force and temperature of cancellated bone and proposed an artificial neural network model to estimate the average value of milling force and temperature [12]. The above studies explored the mechanism of bone tissue device interaction, discussed the thermal damage phenomenon of bone tissue device interaction, and proposed the prediction modeling method and parameter optimization method of bone cutting parameters.

However, most of the above studies focus on the prediction model of cortical bone layer, without modeling the prediction model of cancellous bone and transition area, without considering the influence of different bone density, microstructure, and specific milling trajectory, and lack quantitative analysis of the influence of cutting parameters. At the same time, the parameters obtained and the model established do not consider the lamina milling skills of spinal surgeons and lack the basis of clinical practice.

This paper studies the problems related to robot bone milling operated by a doctor with combining theoretical analysis and experiment. The spinal surgery cooperative robot for lamina milling was operated by a doctor to measure the parameters of lamina milling and analyze their laws and effects when the medical ball end milling cutter was used. Based on the experiment, the prediction model considering the doctor's surgical skills was established and the sensitivity analysis of the effective parameters was carried out. The main study in this study is summarized as follows:

(1) In order to obtain the optimal milling parameters of bone and avoid the bone damage caused by robot milling, the influence of individual differences of bone density, microstructure characteristics, and milling methods should be considered

(2) The empirical prediction model of robot bone milling temperature was established in different bone regions (cortical and cancellated bone regions)

(3) The effect of effective robot bone milling parameters on the output milling temperature was quantitatively studied to discuss the parameter sensitivity

(4) From exploring lamina milling skills of spinal surgery cooperative robot operated by a doctor, the measurement and law research of lamina milling parameters are carried out in the process of doctor operating robot surgery and a reasonable physical description and expression among robot lamina milling skill learning parameters are established to establish a theoretical basis for safety interaction and dynamic control of robot lamina milling

\section{Experimental Method of Bone Milling}

2.1. Robot Bone Milling System. The experimental system of bone milling was established. The experimental device consists of a six-axis cooperative robot, a force sensor, a thermocouple thermometer, and a milling tool. The robot was used in the bone milling experiment. The robot has a payload of $6 \mathrm{~kg}$, a maximum working radius of $914 \mathrm{~mm}$, and a maximum moving speed of $2800 \mathrm{~mm} / \mathrm{s}$. The K-type thermocouple was used to complete bone milling temperature measurement with embedded in the bone samples, whose range is $-50 \sim 1300^{\circ} \mathrm{C}$ and resolution is $0.1^{\circ} \mathrm{C}$. The milling tool adopts a professional spherical milling cutter with a cemented carbide material ball head diameter of $4 \mathrm{~mm}$ for bone resection. The experimental system of robot bone milling is shown in Figure 1.

2.2. Preparation of Bone Specimen. According to the density and structure, the bone is mainly divided into cancellous bone and cortical bone [13], as shown in Figure 2. Cancellous bone structure is composed of trabeculae and pores, in which trabeculae play a supporting role and pores are filled with bone marrow. There is a very thin transition between the cortical bone and cancellous bone, which is called inner circumferential lamellae. The cortical bone is located in the outer layer of bone, which is composed of osteons and bone matrix. Osteons are parallelly arranged and buried in the matrix, and osteons are arranged on the surface of bone as the outer circumferential lamellae. Therefore, the structure of cortical bone makes the bone material anisotropic, that is, it has different thermodynamic properties in different directions. Because the interface strength between the bone and bone plate is relatively weak, it may lead to cracking during cutting. In bone cutting, the formation of bone milling debris is similar to that of unidirectional composites, which mainly depends on the cutting depth and osteon orientation [14], as shown in Figure 3.

Pig femur was used as the experimental material in this paper. The pig femur was processed into a number of cortical 


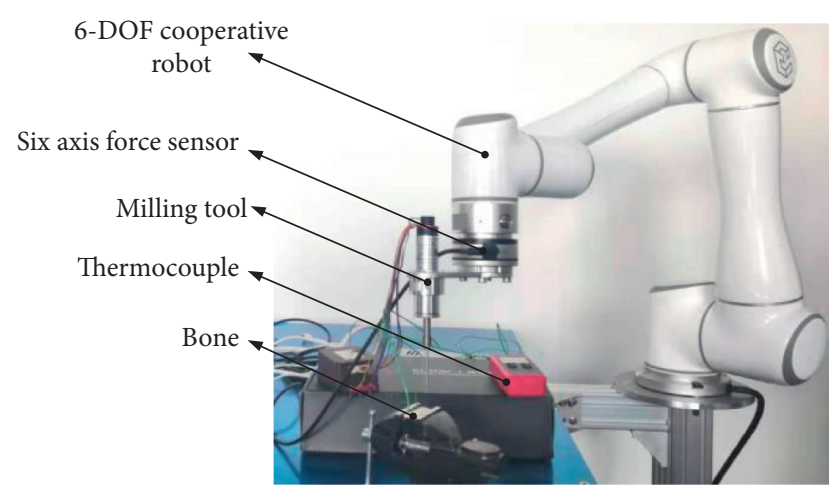

FIgURE 1: Robot bone milling experiment system.

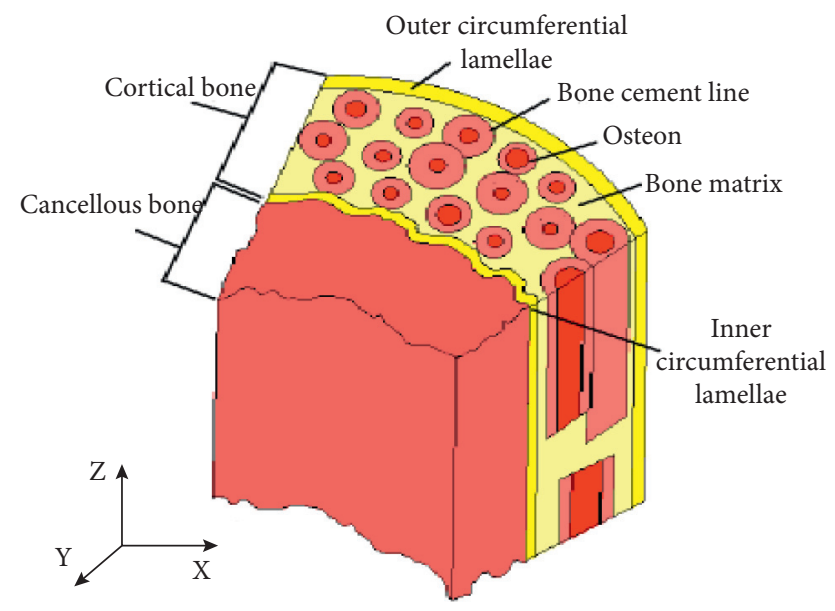

FIgURE 2: Microstructure of the bone.

and cancellous bone blocks with a length of $20 \mathrm{~mm}$, a width of $20 \mathrm{~mm}$, and a height of $6 \mathrm{~mm}$. According to the bone structural characteristics, the cortical bone part can be divided into three directions such as parallel direction, cross direction, and vertical direction relative to the pig femur axis in Figure 4. A thermocouple was placed inside the cutting area perpendicular to the feed direction of a bone block so as to obtain the real-time temperature of bone milling and take the average value of the highest temperature as the final temperature data.

Milling experiments were carried out according to preset experimental sequence. Under different cutting parameters (rotation speed, feed speed and, milling depth), a series of milling experiments were carried out in three cutting modes (vertical cutting mode, parallel cutting mode and cross cutting mode) in the cortical bone area and cancellous bone area. The temperature during the process of bone milling was measured by using a thermocouple; each group of experiment values was filled in Table 1 to explore the influence of rotation speed, feed speed, and milling depth on milling temperature.

2.3. Robot Bone Milling Progress. The cooperative robot emphasizes the dominant position of doctors in the surgical process and uses force (tactile) feedback means to enable doctors and robots to jointly control surgical tools to complete the surgical process, which maintains doctors' working habits, and enable doctors to work according to conventional surgical skills. The process of lamina milling of spinal surgeons has strict specifications, and spinal surgeons have received strict surgical skill training. In order to make the spinal surgery robot reach the skill level of doctors and improve the flexibility and safety of lamina milling of spinal surgery robot, the spinal surgery cooperative robot for lamina milling is operated by a doctor to measure the parameters of lamina milling and analyze their laws and effects, and the reasonable physical description and expression among the parameters of robot lamina milling skills are established, which can establish a theoretical basis for the safe interaction and dynamic control of robot lamina milling.

In this study, doctors operate the spinal surgery cooperative robot for lamina milling, mainly use the cooperative motion of hand and arm to control the mechanical arm, and perceive the surgical operation with the help of human vision, hearing, touch, and motion sense. At the same time the tissue instrument action parameters of the end surgical tools during the doctor's operation are continuously recorded, and the milling temperature and other parameters at different speeds, feed depth, and milling speed are extracted by stages to establish the empirical prediction model of milling skill parameters by stages. On this basis, the influence of input milling skill parameters on output parameters is clarified and quantitatively analyzed, and the best milling skill parameters are found through multiobjective optimization.

2.4. Measurement of Bone Milling Temperature. The working principle diagram of the thermocouple thermometer used in this paper is shown in Figure 5. In order to ensure the accuracy of temperature measurement data, the temperature values of T1 and T2 of the working end 1 and reference end 2 , respectively, need to be first calibrated with a constant temperature bath. The temperature of the constant temperature bath can be set to $45^{\circ} \mathrm{C}, 55^{\circ} \mathrm{C}, 65^{\circ} \mathrm{C}, 75^{\circ} \mathrm{C}, 85^{\circ} \mathrm{C}$, and $98^{\circ} \mathrm{C}$, and reach the set temperature after a period of time. Insert the two ends of the thermocouple thermometer into the same position in the water at the same time, read the temperature values of $\mathrm{T} 1$ and $\mathrm{T} 2$, and calculate the difference between $\mathrm{T} 1$ and $\mathrm{T} 2$ and the maximum error between the set value and the measured value. The calibration experiment data sheet is shown in Table 2. It can be seen that the displayed values of $\mathrm{T} 1$ and $\mathrm{T} 2$ are close to the set temperature, the maximum error is $0.2^{\circ} \mathrm{C}$, and the maximum difference between $\mathrm{T} 1$ and $\mathrm{T} 2$ is $0.3^{\circ} \mathrm{C}$. The difference is less than $1^{\circ} \mathrm{C}$, indicating that the accuracy of the thermocouple thermometer is high and meets the experimental requirements.

In order to obtain the real bone milling temperature required to establish the prediction model, the robot uses a cemented carbide ball end milling cutter to carry out orthogonal cutting experiments and thermocouples to collect the experimental data of bone milling temperature under 


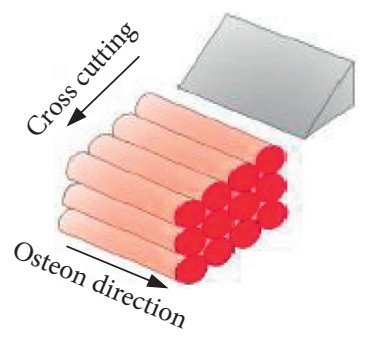

(a)

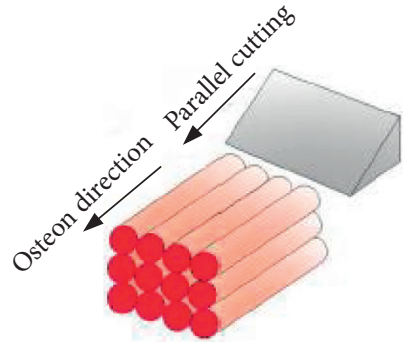

(b)

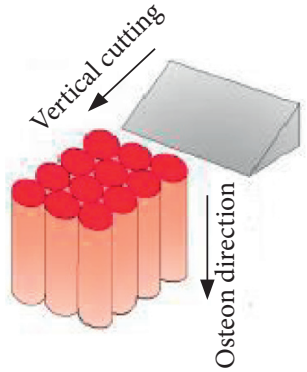

(c)

Figure 3: Three cutting modes: (a) cross cutting mode, (b) parallel cutting mode, and (c) vertical cutting mode.

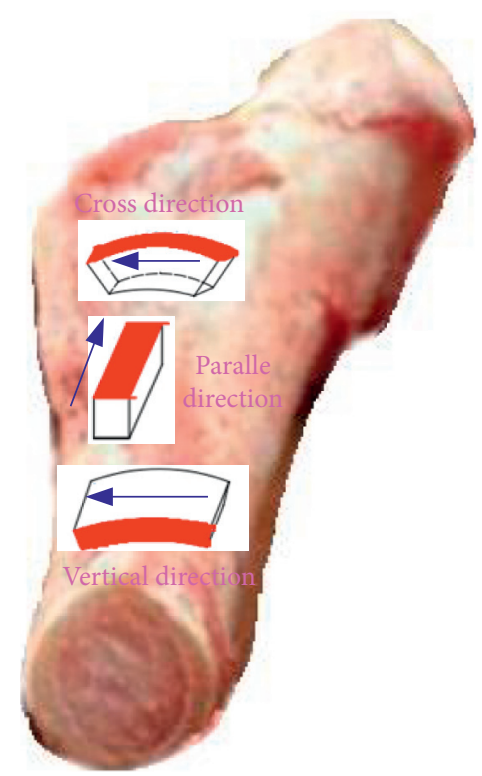

FIGURE 4: Cortical bone part and three cutting directions.

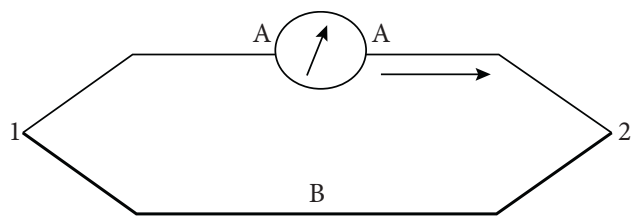

FIGURE 5: Working principle of the thermocouple thermometer.

different cutting modes and different cutting parameters. First, the surface of the bone sample completes one cutting to obtain a flat cutting plane, so as to control the cutting depth in the bone milling experiment, and the thermocouple is inserted and fixed on the surface of the bone sample, so that the thermocouple is cut off when the tool cuts through the temperature measuring point in the actual cutting process, so as to obtain the real instantaneous cutting temperature. As shown in Figure 6, the installation holes are drilled at three different positions in the milling direction on the bone block workpiece to prepare for the subsequent installation of thermocouples. Second, fix the prepared bone samples on the test bench and install thermocouples, accurately adjust the position relationship between the tool and the bone, observe the whole milling process and the surface state of bone milling, measure the change of cutting temperature with time, carry out milling experiments with different milling parameters in different areas, and collect and record the milling temperature results in real time. Figure 7 shows the thermocouple temperature measurement. The ball end milling cutter blade cuts the thermocouple during a single pass through the bone milling surface. The peak value of the pulse in Figure 8 represents the maximum temperature of the bone milling surface measured by the thermocouple. This method can estimate the actual internal temperature of the cutting point.

2.5. Response Surface Method. The response surface method consists of two parts: experimental design and functional fitting, which determine the fitting function accuracy. When using the response surface method to discuss the mathematical relationship between design variables and responses, the factors and levels need to determined first. The number of levels is 2 , while the number of factors generally does not exceed 4 . All these factors are measured data. Next, select the experimental design method, create the experimental design, and determine the test operation sequence. Then, the experiment results are obtained and the experimental data are analyzed. Finally, the selected model is fitted and the model validity is analyzed. The undetermined coefficients of the linear polynomials are determined by the experimental design to fit the response relationship. Let the variable $y$ be a function of $x_{1}, x_{2}, \ldots, x_{n}$, which is expressed as follows:

$$
y=f\left(x_{1}, x_{2}, \ldots, x_{n}\right)+\varepsilon,
$$

where $f\left(x_{1}, x_{2}, \ldots, x_{n}\right)$ is the response coefficient of factor $x_{1}, x_{2}, \ldots, x_{n}$ and $\varepsilon$ is the random error. It can be seen from the mathematical method that

$$
E_{y}=f\left(x_{1}, x_{2}, \ldots, x_{n}\right) .
$$

If the relation between $E_{y}=f\left(x_{1}, x_{2}, \ldots, x_{n}\right)$ is known, the relation between $y$ and $x_{1}, x_{2}, \ldots, x_{n}$ can be found. In order to find the optimal combination of research objectives more conveniently, a reasonable model is needed to reflect the research objectives. In general, a second-order model can meet the research requirement, and the expression is shown as follows: 
TABLe 1: Experiment sequence and data results.

\begin{tabular}{|c|c|c|c|c|c|c|c|c|}
\hline Serial number & Order & $A(\mathrm{r} / \mathrm{min})$ & $B(\mathrm{~mm} / \mathrm{s})$ & $C(\mathrm{~mm})$ & $T_{1}\left({ }^{\circ} \mathrm{C}\right)$ & $T_{2}\left({ }^{\circ} \mathrm{C}\right)$ & $T_{3}\left({ }^{\circ} \mathrm{C}\right)$ & $T_{4}\left({ }^{\circ} \mathrm{C}\right)$ \\
\hline 8 & 2 & 3000 & 120 & 0.3 & 43.2 & 46 & 47.6 & 41 \\
\hline 12 & 3 & 2000 & 120 & 0.2 & 35.4 & 38.6 & 40 & 33.1 \\
\hline 19 & 4 & 2000 & 75 & 0.2 & 37 & 40.8 & 42.1 & 35 \\
\hline 6 & 5 & 3000 & 30 & 0.3 & 49 & 51.7 & 53 & 46.9 \\
\hline 11 & 6 & 2000 & 30 & 0.2 & 41.2 & 44 & 45.7 & 39 \\
\hline 10 & 7 & 3000 & 75 & 0.2 & 42 & 45.2 & 46.6 & 39.7 \\
\hline 3 & 8 & 1000 & 120 & 0.1 & 23 & 26.1 & 27.9 & 21 \\
\hline 2 & 9 & 3000 & 30 & 0.1 & 45.1 & 48 & 49.3 & 43 \\
\hline 17 & 10 & 2000 & 75 & 0.2 & 37.2 & 40.3 & 41.9 & 35.1 \\
\hline 13 & 11 & 2000 & 75 & 0.1 & 36.1 & 39 & 41 & 33.8 \\
\hline 9 & 12 & 1000 & 75 & 0.2 & 25.8 & 29 & 30.4 & 23.6 \\
\hline 20 & 13 & 2000 & 75 & 0.2 & 36.8 & 39.8 & 41.5 & 34.6 \\
\hline 18 & 14 & 2000 & 75 & 0.2 & 37.1 & 40 & 41.6 & 35 \\
\hline 5 & 15 & 1000 & 30 & 0.3 & 32.9 & 36.1 & 37.6 & 30.7 \\
\hline 14 & 16 & 2000 & 75 & 0.3 & 39.8 & 43 & 44.2 & 37.5 \\
\hline 7 & 17 & 1000 & 120 & 0.3 & 27 & 30 & 31.6 & 24.9 \\
\hline 15 & 18 & 2000 & 75 & 0.2 & 37 & 40 & 41.3 & 34.8 \\
\hline 16 & 19 & 2000 & 75 & 0.2 & 37.1 & 40.2 & 41.7 & 35 \\
\hline 1 & 20 & 1000 & 30 & 0.1 & 29 & 31.3 & 32.8 & 27 \\
\hline
\end{tabular}

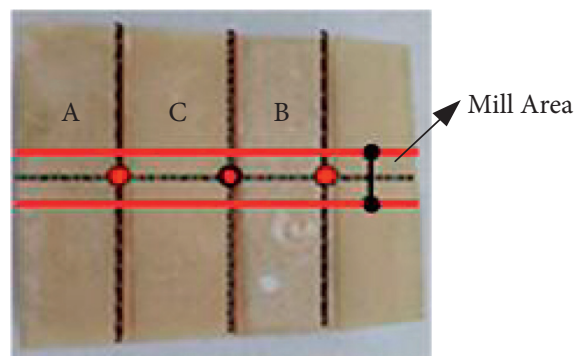

FIGURE 6: Bone drilling for thermocouple installation.

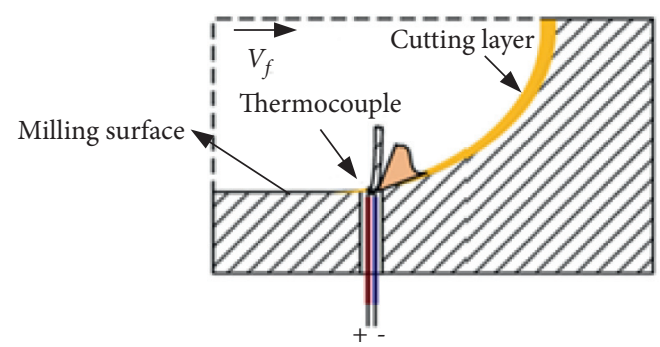

Figure 7: Thermocouple temperature measurement.

$$
y=\beta_{0}+\sum_{i=1}^{k} \beta_{i} x_{i}+\sum_{i=1}^{k} \beta_{i i} x_{i}^{2}+\sum_{i} \sum_{j} \beta_{i j} x_{i} x_{j}+\varepsilon,
$$

where $y$ is the response function; $\beta$ is the regression coefficient matrix; $\beta_{i}, \beta_{i i}$, and $\beta_{i j}$ are the parameter coefficients of linear, square, and interaction factor effects, respectively; $x_{i}$ and $x_{j}$ represent all the influencing factors; and $\varepsilon$ is the error term. The second-order response model considers the influence of single factor and its secondary effects and interaction effects on the response, so it can predict the response more effectively. Finally, ANOVA can be used to verify the model.

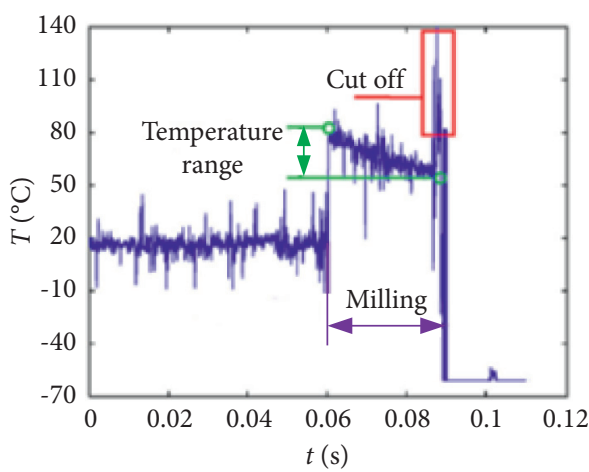

Figure 8: Temperature signal measured by using a thermocouple.

2.6. Central Composite Design Method. In this study, central composite design was used to design the experimental scheme. As shown in Figure 9, three types of test points (cubic points, axial points, and center points) are usually selected in the central composite design in the experiment space. The specific test points are composed as follows:

$$
N=m_{c}+m_{r}+m_{o},
$$

where $m_{c}$ represents the cubic point. If there are $k$ impact factors, each factor is taken as two levels $(-1,1)$, then there are $2^{k}$ test points in $m_{c} . m_{r}$ represents the axial point, which is distributed in the axial direction. If there are $k$ impact factors, there are a total of $2^{k}$ test points in $m_{r} . m_{o}$ is the center point, which means the number of times to repeatedly take the zero level. In general, $m_{o} \geq 3$. The distance between the axial point and the center point $(\alpha)$ is called the asterisk arm, and $\alpha$ is an undetermined parameter. Multiple starting points can be used in the selection. If there are $k$ impact factors, $\alpha=2^{k / 4}$ should be set, and the expected experimental function can be obtained by adjusting $\alpha$.

In this study, the maximum cutting force and the highest surface temperature of bone milling area were taken as indexes, 


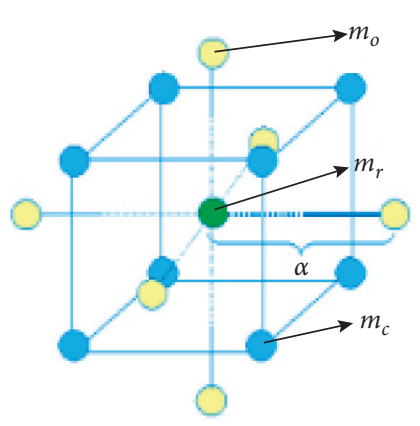

Figure 9: Central composite design diagram.

with the milling parameters (rotation speed $A$, feed speed $B$, and milling depth $C$ ) for the influencing factors, the second-order response surface central composite design was chosen, and the experiment design factor level table was established, where there are three factors, $k=3$, so the cubic point $m_{c}=8$ and the axial point $m_{r}=6 . m_{o}=6$ was chosen as the center point mainly to estimate the experimental error and where $\alpha \approx 1$. Table 3 shows the arrangement of experiment points in the center of composite design.

In this paper, three factors (rotation speed $A$, feed speed $B$, and milling depth $C$ ) affecting the milling force and temperature were mainly studied. Table 4 is the variation level table of milling experiment parameters, and Table 5 is the coding table of the variation range of experiment factors and the level of quadratic regression design factors.

2.7. Sobol Method. The Sobol method is a variance-based sensitivity analysis [15]. The variance-based method depends on the following number of estimates:

$$
S=\frac{V_{x}[E(y \mid x)]}{V(y)}
$$

where $y$ is the output variable, $x$ is the input variable, $E(y \mid x)$ is the expected value of $y$ at a fixed value of $x$, the variance takes all possible values of $x$, and $V(y)$ is the total variance of the output $y$.

Let the model be $y=f(x)\left(x=x_{1}, x_{2}, \ldots, x_{k}\right)$, where $x_{i}$ is uniformly distributed, and $f^{2}(x)$ is integrable in the domain, which can be decomposed into

$$
\begin{aligned}
f(x)= & f_{0}+\sum_{i=1}^{n} f_{i}\left(x_{i}\right)+\sum_{i=1}^{n} f_{i \leq j \leq n}\left(x_{i}, x_{j}\right) \\
& +\cdots+f_{1, \ldots k}\left(x_{i 1}, x_{i 2}, \ldots, x_{i k}\right) .
\end{aligned}
$$

Therefore, based on the Sobol method, the total variance of model $y$ in this study is decomposed into the variance of a single parameter and the variance generated by the interaction between parameters. The total variance $V$ of model $y$ can be decomposed as follows:

$$
V=\sum_{i=1}^{n} V_{i}+\sum_{i \leq j \leq n}^{n} V_{i j}+\cdots+V_{1, \ldots, k},
$$

where $V_{i}$ represents the folk square difference generated by parameter $x_{i}, V_{i j}$ represents the variance of the interaction between two parameters $x_{i}$ and $x_{j}$, and $V_{k}$ represents the variance of $k$ parameters acting together.
TABLE 2: Calibration results of the thermocouple thermometers.

\begin{tabular}{lccccc}
\hline No. & $\begin{array}{c}\text { Temperature } \\
\text { setting }\left({ }^{\circ} \mathrm{C}\right)\end{array}$ & $\begin{array}{c}\mathrm{T} 1 \\
\left({ }^{\circ} \mathrm{C}\right)\end{array}$ & $\begin{array}{c}\mathrm{T} 2 \\
\left({ }^{\circ} \mathrm{C}\right)\end{array}$ & $\begin{array}{c}\mathrm{T} 2-\mathrm{T} 1 \\
\left({ }^{\circ} \mathrm{C}\right)\end{array}$ & $\begin{array}{c}\text { Maximum } \\
\text { error }\left({ }^{\circ} \mathrm{C}\right)\end{array}$ \\
\hline 1 & 45 & 45.1 & 44.8 & 0.3 & 0.2 \\
2 & 55 & 44.9 & 55.0 & 0.1 & 0.1 \\
3 & 65 & 65.2 & 64.8 & 0.4 & 0.2 \\
4 & 75 & 75.1 & 75.2 & 0.1 & 0.2 \\
5 & 85 & 85.0 & 85.1 & 0.1 & 0.1 \\
6 & 98 & 98.1 & 98.0 & 0.1 & 0.1 \\
\hline
\end{tabular}

TABle 3: Arrangement of experiment points for central composite design.

\begin{tabular}{lc}
\hline Item & Number \\
\hline Number of factors $P$ & 3 \\
Cubic point $m_{c}$ & 8 \\
Axial point $m_{r}$ & 6 \\
Center point $m_{o}$ & 6 \\
Center point number (orthogonal) & 9 \\
Total experiment number (uniform precision) & 20 \\
Total experiment number (orthogonal) & 23 \\
\hline
\end{tabular}

The specific steps of Sobol global sensitivity analysis method are as follows: first, the parameters that need sensitivity analysis are selected. In this paper, milling parameters (rotation speed $A$, feed speed $B$, and milling depth $C$ ) of ball milling cutter are presented. Then, the variation range of each parameter is determined. The range of rotation speed $A$ is $1000-3000 \mathrm{r} / \mathrm{min}$, the range of feed speed $B$ is $30-120 \mathrm{~mm} / \mathrm{min}$, and the range of milling depth $C$ is $0.1-0.3 \mathrm{~mm}$. The distribution of each input milling parameter is uniformly distributed. The mean value $f_{0}$ and the total variance $V$ of each parameter are calculated by the Monte Carlo method as follows:

$$
\begin{aligned}
& f_{0}=\int f(x) \mathrm{d} x \approx \frac{1}{N} \sum_{k=1}^{N} f\left(x_{k}\right), \\
& V=\int f^{2}(x) \mathrm{d} x-f_{0}^{2} \approx \frac{1}{N} \sum_{k=1}^{N} f^{2}\left(x_{k}\right)-f_{0}^{2},
\end{aligned}
$$

where the average value $f_{0}$ must be a constant, $N$ is the size of Monte Carlo sampling, and the number of samples in this study was selected as 3000 . By fixing the value of parameters and changing other parameters, the folk square difference or first-order effect $V_{i}$ of each parameter was calculated, that is, the influence degree of a single parameter on the model results was defined as follows:

$$
V_{i}=\int f_{i} d x_{i}
$$

The influence degree of the interaction between parameters on the model results can be expressed by the following equation:

$$
V_{i_{1}, i_{2}, \ldots, i_{k}}=\int f_{i_{1}, i_{2}, \ldots, i_{k}}^{2} \mathrm{~d} x_{i_{1}}, \mathrm{~d} x_{i_{2}}, \ldots, \mathrm{d} x_{i_{k}} .
$$

Let the single parameter of the objective function model and the sensitivity index $S$ of the interaction between the parameters be expressed as: 
TABLE 4: Level changes of milling parameters.

\begin{tabular}{lccc}
\hline$x$ & Under level & Above level & Zero level \\
\hline Rotation speed $A(\mathrm{r} / \mathrm{min})$ & 1000 & 3000 & 2000 \\
Feed speed $B(\mathrm{~mm} / \mathrm{min})$ & 30 & 120 & 75 \\
Milling depth $C(\mathrm{~mm})$ & 0.1 & 0.5 & 0.3 \\
\hline
\end{tabular}

$$
S_{i_{1}, \ldots, i_{s}}=\frac{V_{i_{1}, \ldots, i_{s}}}{V}, \quad 1 \leq i_{1}<\ldots<i_{s} \leq k .
$$

Therefore, for parameter $x_{i}$, the first-order sensitivity index can be obtained as $S_{i}=V_{i} / V$, which measures the main influence of parameter $x_{i}$ on the output model result $y$, that is, the partial contribution of parameter $x_{i}$ to the variance $V(y)$. Similarly, for $i \neq j$, the second-order sensitivity index of $V(y)$ is $S_{i j}=V_{i j} / V$, which measures the influence of the interaction between parameters $x_{i}$ and $x_{j}$ on the output model result $y$.

After normalization of equation (12), the following equation can be obtained:

$$
1=\sum_{i=1}^{N} S_{i}+\sum_{i \leq j \leq n}^{N} S_{i j}+\cdots+S_{1,2, \ldots, k}
$$

The global sensitivity of each input parameter refers to the sensitivity of all the parameters. The global sensitivity definition index can be expressed as follows:

$$
S_{T i}=S_{i}+\sum_{i \neq j} S_{i j}+\cdots
$$

\section{Results and Discussion}

3.1. Prediction Model of Milling Temperature. Based on the central composite experimental design method, the central composite in response surface model was selected for experiment design. The rotation speed $A$, feed speed $B$, and milling depth $C$ are filled in Table 3 , and 20 experiment design points were automatically analyzed and constructed by Design-Expert software. In the cortical bone area, milling experiments were carried out in three cutting directions: parallel cutting direction, cross cutting direction, and vertical cutting direction. The milling temperatures are $T_{1}$ in parallel cutting mode, $T_{2}$ in cross cutting mode, $T_{3}$ in vertical cutting mode, and $T_{4}$ in the cancellous area, respectively. The sequence of experiment points and experiment data results are shown in Table 1.

Considering the influence of bone microstructure and milling trajectory, the empirical mathematical models of milling temperature were established in cortical and cancellous bone segments. The reliability was set as $95 \%$, and $P<0.05$ was the level of statistical significance. The variance analysis of effective parameters was used to compare the differences of different parameters and combinations to verify the validity of different model items of milling parameters. The prediction model of the maximum milling temperatures $T_{1}, T_{2}$, and $T_{3}$ under parallel cutting mode, cross cutting mode, and vertical cutting mode in the cortical bone region and the prediction model of milling temperature $T_{4}$ in cancellous bone regions are shown as follows:

$$
\begin{aligned}
T_{1}= & 16.605+0.021 A-0.16 B-17.452 C-8.471 \times 10^{-2} A B+2.5 \times 10^{-4} A C \\
& +0.011 B C-3.155 \times 10^{-6} A^{2}+6.15 B^{2}+89.545 C^{2}, \\
T_{2}= & 17.333+0.021 A-0.137 B-6.224 C-5.555 \times 10^{-7} A B-1.5 \times 10^{-3} A C \\
& -0.022 B C-3.123 \times 10^{-6} A^{2}+5.32 \times 10^{-4} B^{2}+77.727 C^{2}, \\
T_{3}= & 19.2+0.021 A+0.026 B-0.138 C-2.777 \times 10^{-7} A B-1.125 \times 10^{-3} A C \\
& -0.025 B C-3.236 \times 10^{-6} A^{2}+5.499 \times 10^{-4} B^{2}+86.364 C^{2}, \\
T_{4}= & 16.605+0.021 A-0.16 B-17.452 C-8.471 \times 10^{-2} A B+2.5 \times 10^{-4} A C \\
& +0.011 B C-3.155 \times 10^{-6} A^{2}+6.15 B^{2}+89.545 C^{2},
\end{aligned}
$$

where $A$ represents rotation speed, $B$ represents feed speed, and $C$ represents milling depth.

3.2. Prediction Result Analysis. Milling temperature $T_{1}$ in the parallel cutting mode, temperature $T_{2}$ in the cross cutting mode, milling temperature $T_{3}$ in the vertical cutting mode were collected, respectively, and then milling temperature $T_{4}$ was collected in cancellous bone region. Table 6 shows the results of variance analysis of quadratic regression equation prediction model of milling temperature under the parallel cutting mode of cortical bone area by ANOVA module, where the unimportant model items were eliminated. It can be seen from Table 6 that, in the influence factors of the model, rotation speed $A$ and feed speed $B$ are significant, milling depth $C$ is not significant, and quadratic terms such 
TABLE 5: Range of test factors and coding table of quadratic regression design.

\begin{tabular}{|c|c|c|c|c|c|c|}
\hline \multirow{2}{*}{$\begin{array}{l}\text { Range of variable } x \\
\text { Variable }\end{array}$} & \multicolumn{3}{|c|}{ Actual variable $x$} & \multicolumn{3}{|c|}{ Standardization of $z$} \\
\hline & $x_{1}$ & $x_{2}$ & $x_{3}$ & $z_{1}$ & $z_{2}$ & $\overline{z_{3}}$ \\
\hline Above level & $x_{12}$ & $x_{22}$ & $x_{32}$ & 1 & 1 & 1 \\
\hline Zero level & $x_{10}$ & $x_{20}$ & $x_{30}$ & 0 & 0 & \\
\hline Under level & $x_{11}$ & $x_{21}$ & $x_{31}$ & -1 & -1 & \\
\hline Distance from $r$ horizontal & $r \times \Delta_{1}+x_{10}$ & $r \times \Delta_{2}+x_{20}$ & $r \times \Delta_{3}+x_{30}$ & $r$ & $r$ & $r$ \\
\hline $\begin{array}{l}\text { Distance from }-r \text { horizontal } \\
\text { Standard deviation }\end{array}$ & $\begin{array}{c}r \times \Delta_{1}+x_{10} \\
\Delta_{1}\end{array}$ & $\begin{array}{c}r \times \Delta_{2}+x_{20} \\
\Delta_{2}\end{array}$ & $\begin{array}{c}r \times \Delta_{3}+x_{30} \\
\Delta_{3}\end{array}$ & $-r$ & $-r$ & $-r$ \\
\hline
\end{tabular}

TABle 6: Analysis of variance for quadratic regression equation $T_{1}$.

\begin{tabular}{lcccccc}
\hline Factors & Sum of squares of deviations & Degrees of freedom & Mean square error & $F$ & $P($ Prob $>F)$ \\
\hline Model & 803.78 & 9 & 89.31 & 6001.18 & 0.0201 & Significant \\
Rotation speed $A$ & 649.64 & 1 & 649.64 & 43653.00 & 0.0098 & Significant \\
Feed speed $B$ & 87.62 & 1 & 87.62 & 5887.45 & 0.0054 & Significant \\
Milling depth $C$ & 38.81 & 1 & 38.81 & 2607.81 & 0.0136 & Significant \\
$A B$ & 0.000 & 1 & 0.000 & 0.000 & 1.0000 \\
$A C$ & $5.000 E-003$ & 1 & $5.000 E-003$ & 0.34 & 0.5750 \\
$B C$ & 0.020 & 1 & 0.020 & 1.34 & 0.2733 \\
$A^{2}$ & 27.37 & 1 & 27.37 & 1838.87 & $<0.0001$ \\
$B^{2}$ & 4.27 & 1 & 4.27 & 286.64 & $<0.0001$ \\
$C^{2}$ & 2.21 & 1 & 2.21 & 148.17 & $<0.0001$ \\
Residual & 0.15 & 10 & 0.015 & & \\
Misfit term & 0.055 & 5 & 0.011 & 0.59 & 0.7090 \\
Pure error & 0.093 & 5 & 0.019 & & Not significant \\
\hline
\end{tabular}

as $A B, A C, B C, A^{2}, B^{2}$, and $C^{2}$ are not significant. The misfit term in the table is used to indicate the degree of difference between the model and the experiment. In this fitting, $P>0.05$, the misfit term is not significant, which is beneficial to the model, and there is no misfit factors, indicating that the prediction model can replace the simulation point to analyze the simulation results. It can be seen from Table 6 that the fitting effect of quadratic multinomial model is significant and rotation speed $A$ and feed speed $B$ are the significant factors influencing milling temperature, and rotation speed $A$ has the most significant influence.

Figure 10(a) shows the normal probability distribution of residual errors in the prediction model of bone milling temperature $T_{1}$, which is most in line with the ideal situation. As can be seen from the figure, the points are all distributed near the straight line. Figure 10(b) shows the relationship between the predicted value and the actual value of the model. The closer the point is to the straight line, the smaller the difference between the actual simulation value and the predicted value of the model. According to the above analysis, the prediction models constructed are all reasonable.

Figure 11 shows the three-dimensional response curves of the influence of milling parameters on temperature $T_{1}$. It can be clearly seen from Figure 11(a) that, within the range of study parameters, the milling temperature will increase with the increase of rotating speed and decrease with the increase of feeding speed. It can be concluded that the highest region of milling temperature is concentrated in the region with high rotational speed and low feed speed. It can be seen from Figure 11(b) that, within the range of study parameters, the milling temperature increases with the increase of rotating speed and milling depth. Therefore, when the milling temperature is large, both the rotation speed and the milling depth are large. It can be seen from Figure 11(c) that, within the range of study parameters, the milling temperature decreases with the increase of feed speed and increases with the increase of milling depth. Therefore, the larger area of milling temperature is concentrated when the feed speed is smaller and the milling depth is larger.

Table 7 shows the results of analysis of variance (ANOVA) for the prediction model of milling temperature $\mathrm{T}_{2}$ in the cross cutting mode of cortical bone region, respectively.

Figure 12(a) shows the normal probability distribution of residual errors in the prediction model of bone milling temperature $T_{2}$, which is most in line with the ideal situation. As can be seen from the figure, the points are all distributed near the straight line. Figure 12(b) shows the relationship between the predicted value and the actual value of the model. The closer the point is to the straight line, the smaller the difference between the actual simulation value and the predicted value of the model. According to the above analysis, the prediction models constructed are all reasonable.

Figure 13 shows the three-dimensional response curves of the influence of milling parameters on temperature $T_{2}$. It can be clearly seen from Figure 12(a) that, within the range of study parameters, the effects of rotational speed and feed speed on milling temperature $T_{2}$ in the cross mode are similar to those in the parallel cutting mode. The milling temperature will increase with the increase of rotating speed 


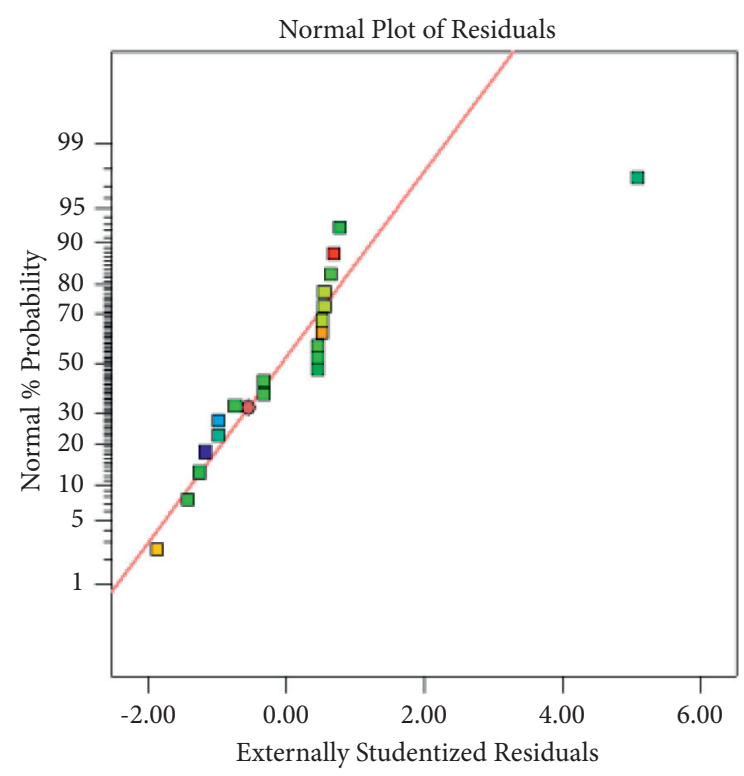

(a)

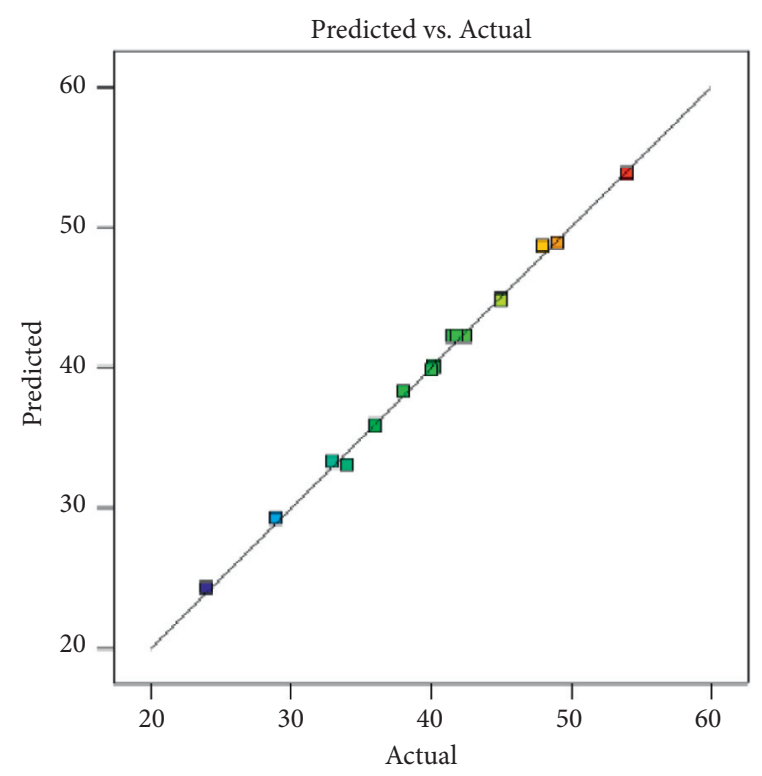

(b)

Figure 10: Residual analysis of prediction equation $T_{1}$. (a) Residual normal probability distribution and (b) relationship between the predicted value and the actual value.

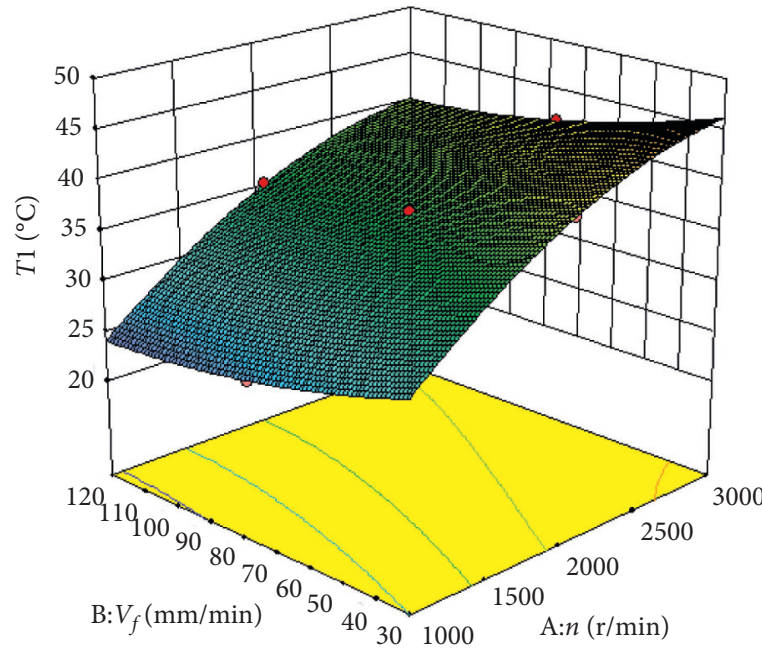

(a)

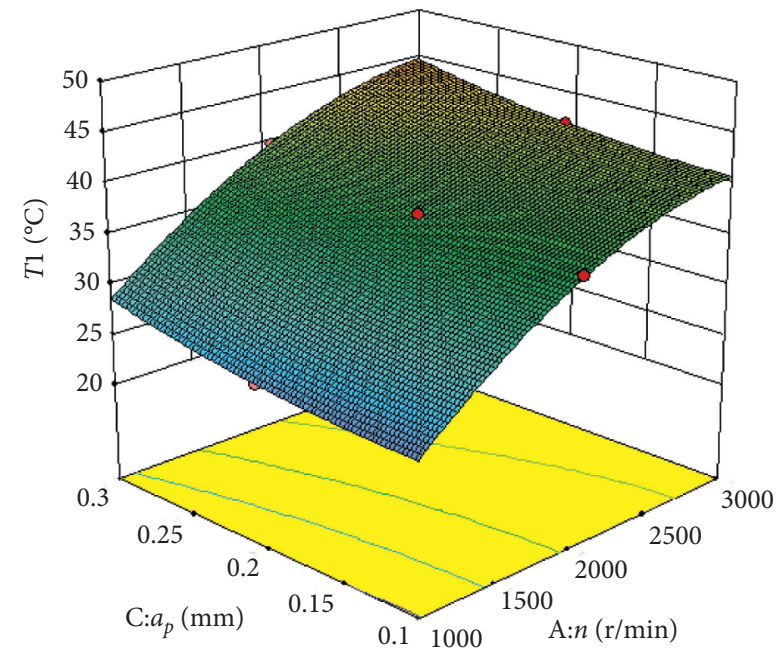

(b)

Figure 11: Continued. 


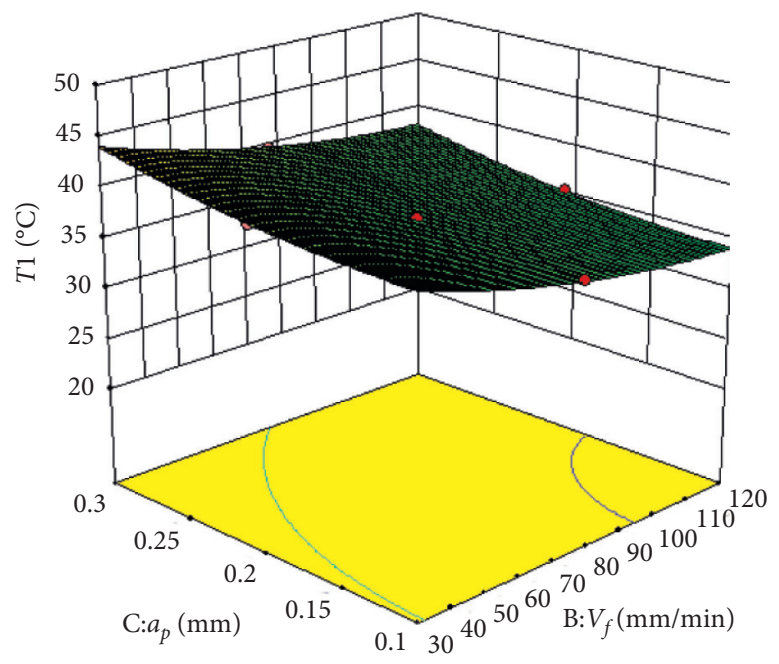

(c)

FIgURE 11: Response surface curves of the influence of milling parameters on $T_{1}$. (a) Effect of rotation speed $A$ and feed rate $B$ on $T_{1}$, (b) effect of rotation speed $A$ and milling depth $C$ on $T_{1}$, and (c) effect of feed speed $B$ and milling depth $C$ on $T_{1}$.

TABLe 7: Analysis of variance for quadratic regression equation $T_{2}$.

\begin{tabular}{|c|c|c|c|c|c|c|}
\hline Factors & Sum of squares of deviations & Degrees of freedom & Mean square error & $F$ & $P($ Prob $>F)$ & \\
\hline Model & 798.09 & 9 & 88.68 & 1137.67 & 0.0361 & Significant \\
\hline Rotation speed $A$ & 649.64 & 1 & 649.64 & 8334.49 & 0.0081 & Significant \\
\hline Feed speed $B$ & 79.52 & 1 & 79.52 & 1020.25 & 0.0324 & Significant \\
\hline Milling depth $C$ & 40.80 & 1 & 40.80 & 523.49 & 0.0101 & Significant \\
\hline$A B$ & $5.000 E-003$ & 1 & $5.000 E-003$ & 0.064 & 0.4933 & \\
\hline$A C$ & 0.18 & 1 & 0.18 & 2.31 & 0.2976 & \\
\hline$B C$ & 0.080 & 1 & 0.080 & 1.03 & 0.5736 & \\
\hline$A^{2}$ & 26.82 & 1 & 26.82 & 344.04 & 0.0004 & \\
\hline$B^{2}$ & 3.19 & 1 & 3.19 & 40.94 & 0.0007 & \\
\hline$C^{2}$ & 1.66 & 1 & 1.66 & 21.32 & 0.1472 & \\
\hline Residual & 0.78 & 10 & 0.078 & & & \\
\hline Misfit term & 0.17 & 5 & 0.034 & 0.28 & 0.4084 & Significant \\
\hline Pure error & 0.61 & 5 & 0.12 & & & \\
\hline
\end{tabular}

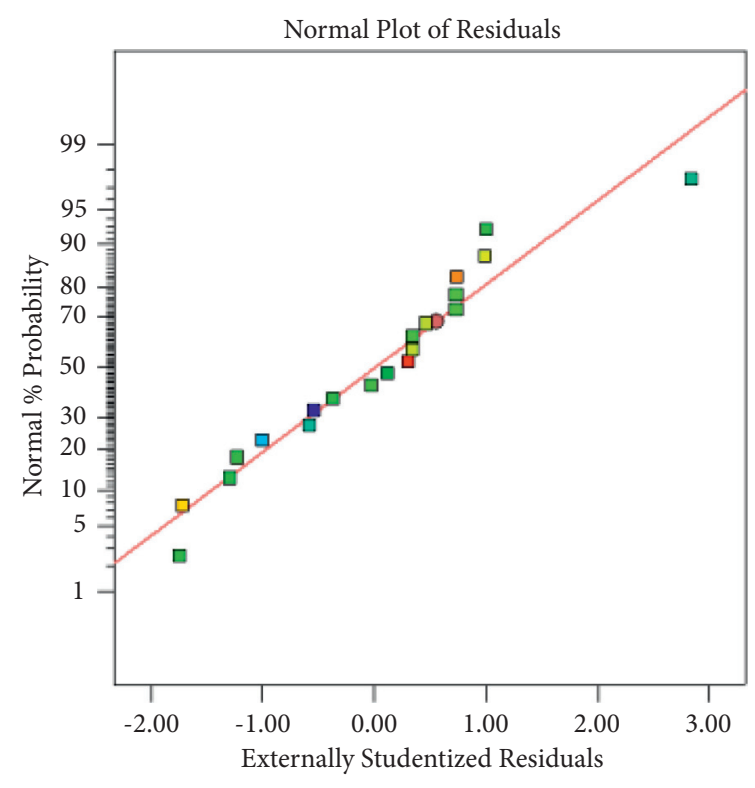

(a)

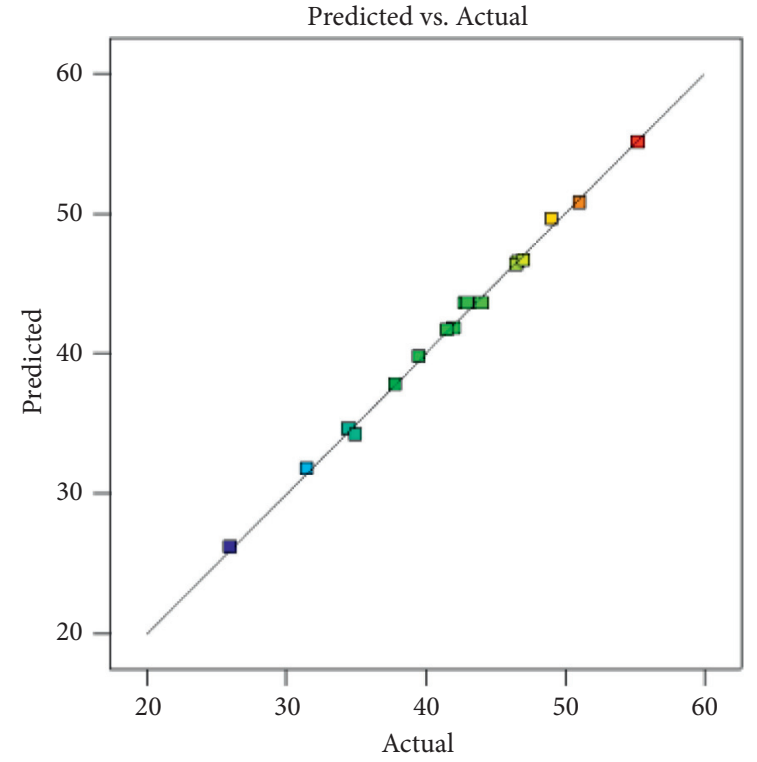

(b)

Figure 12: Residual analysis of prediction equation $T_{2}$. (a) Residual normal probability distribution and (b) relationship between the predicted value and actual value. 


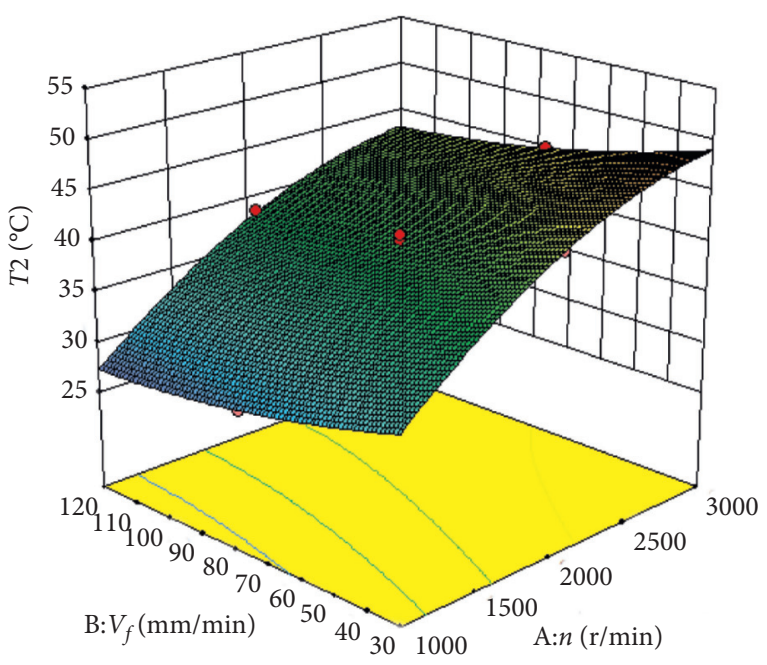

(a)

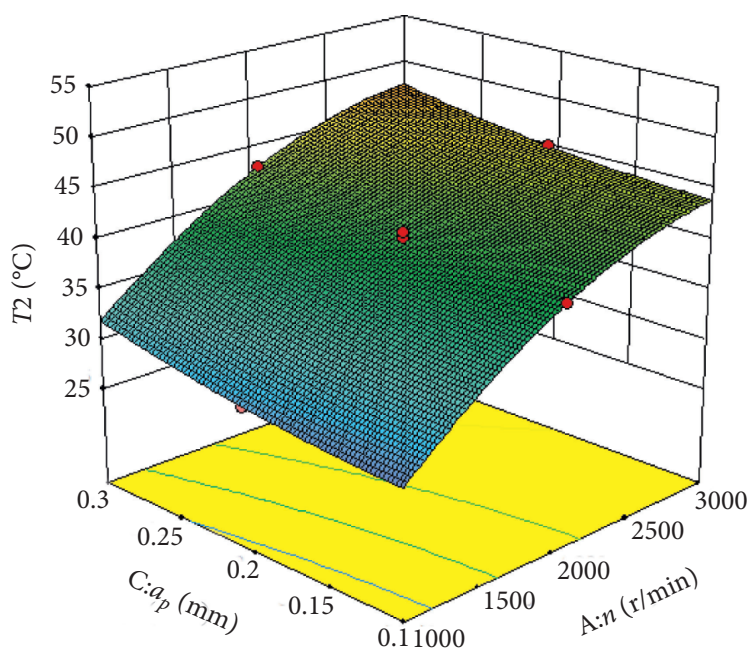

(b)

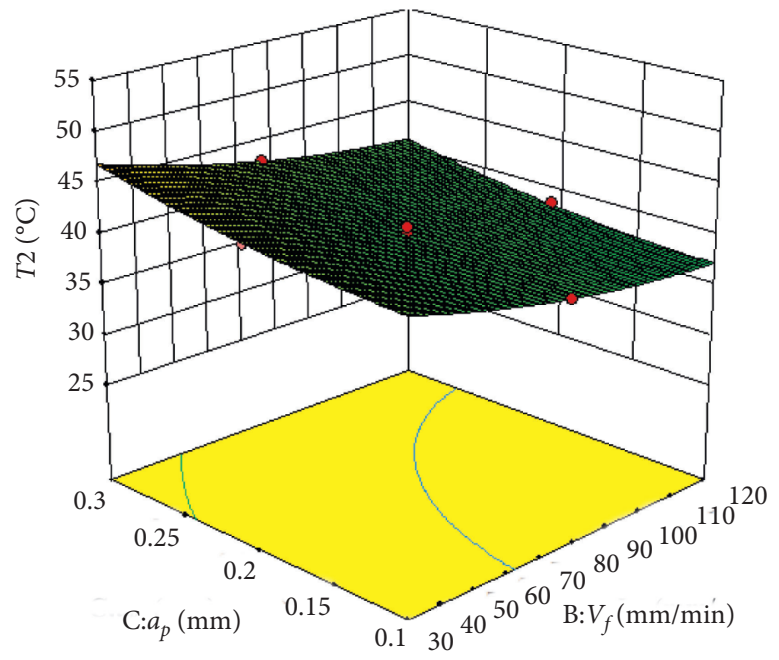

(c)

FIgURE 13: Response surface curves of the influence of milling parameters on $T_{2}$. (a) Effect of rotation speed $A$ and feed speed $B$ on $T_{2}$, (b) effect of rotation speed $A$ and milling depth $C$ on $T_{2}$, and (c) effect of feed speed $B$ and milling depth $C$ on $T_{2}$.

and decrease with the increase of feeding speed. The highest area of milling temperature is concentrated in the case of high rotating speed and low feeding speed. It can be seen from Figure 13(b) that, within the range of study parameters, the milling temperature increases with the increase of rotating speed and milling depth. Therefore, when the milling temperature is large, both the rotation speed and the milling depth are large. It can be seen from Figure 13(c) that, within the range of the study parameters, the milling temperature decreases with the increase of feed speed and increases with the increase of milling depth. Therefore, the larger area of milling temperature is concentrated when the feed speed is smaller and the milling depth is larger.

Table 8 shows the results of variance analysis of the prediction model of the quadratic regression equation of temperature $T_{3}$ in the vertical cutting mode of cortical bone region by using ANOVA module. Figure 14(a) shows the normal probability distribution of residual errors in the prediction model of bone milling temperature $T_{3}$, which is most in line with the ideal situation. As can be seen from the figure, the points are all distributed near the straight line. Figure 14(b) shows the relationship between the predicted value and the actual value of the model. The closer the point is to the straight line, the smaller the difference between the actual simulation value and the predicted value of the model. According to the above analysis, the prediction models constructed are all reasonable.

Figure 15 shows the three-dimensional response curves of the influence of milling parameters on temperature $T_{3}$. It can be clearly seen from Figure 15(a) that, within the range of study parameters, the milling temperature will increase with the increase of rotating speed and decrease with the increase of feeding speed. The highest area of milling temperature is concentrated in the case of high rotating speed and low feeding speed. It can be seen from Figure 15(b) that, within the range of study parameters, the milling temperature increases with the increase of rotating speed and milling depth. Therefore, when the milling 
TABLE 8: Analysis of variance for quadratic regression equation $T_{3}$.

\begin{tabular}{|c|c|c|c|c|c|c|}
\hline Factors & Sum of squares of deviations & Degrees of freedom & Mean square error & $F$ & $P($ Prob $>F)$ & \\
\hline Model & 781.71 & 9 & 86.86 & 912.73 & 0.0351 & Significant \\
\hline Rotation speed $A$ & 638.40 & 1 & 638.40 & 6708.62 & 0.0106 & Significant \\
\hline Feed speed $B$ & 76.18 & 1 & 76.18 & 800.49 & 0.0091 & Significant \\
\hline Milling depth $C$ & 37.25 & 1 & 37.25 & 391.43 & 0.0263 & Significant \\
\hline$A B$ & $1.250 E-003$ & 1 & $1.250 E-003$ & 0.013 & 0.9110 & \\
\hline$A C$ & 0.10 & 1 & 0.10 & 1.06 & 0.3266 & \\
\hline$B C$ & 0.10 & 1 & 0.10 & 1.06 & 0.3266 & \\
\hline$A^{2}$ & 28.80 & 1 & 28.80 & 302.68 & $<0.0001$ & \\
\hline$B^{2}$ & 3.41 & 1 & 3.41 & 35.84 & 0.0001 & \\
\hline$C^{2}$ & 2.05 & 1 & 2.05 & 21.55 & 0.0009 & \\
\hline Residual & 0.95 & 10 & 0.095 & & & \\
\hline Misfit term & 0.54 & 5 & 0.11 & 1.33 & 0.3808 & Not significant \\
\hline Pure error & 0.41 & 5 & 0.082 & & & \\
\hline
\end{tabular}

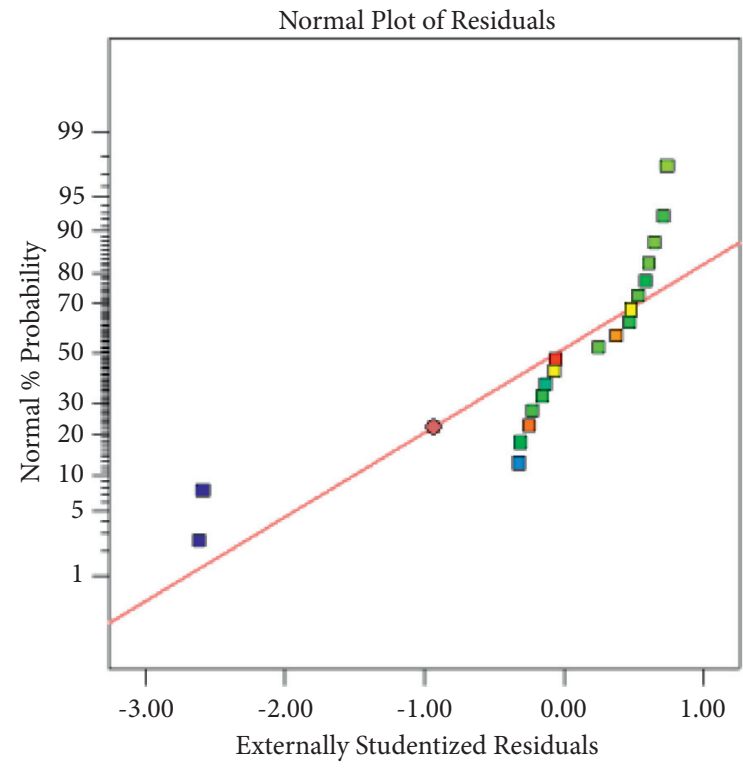

(a)

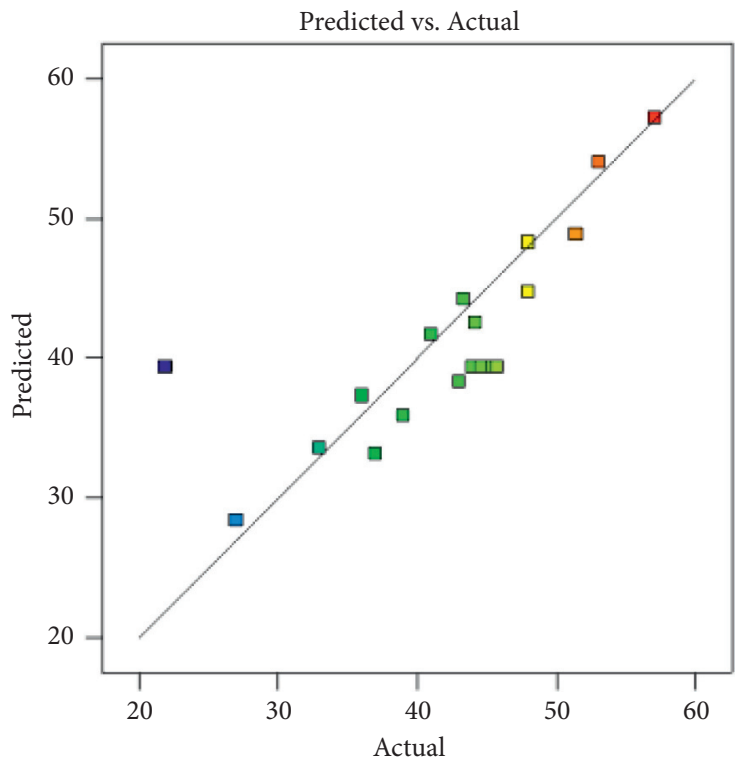

(b)

FIgURE 14: Residual analysis of prediction equation $T_{3}$. (a) Residual normal probability distribution and (b) relationship between the predicted value and the actual value.

temperature is large, both the rotation speed and the milling depth are large. It can be seen from Figure 15(c), within the range of the study parameters, the milling temperature decreases with the increase of feed speed and increases with the increase of milling depth. Therefore, the larger area of milling temperature is concentrated when the feed speed is smaller and the milling depth is larger.

Table 9 shows the results of variance analysis of the prediction model of the quadratic regression equation of milling temperature $T_{4}$ in the cancellous bone region by using ANOVA module. Figure 16(a) shows the normal probability distribution of residual errors in the prediction model of bone milling temperature $T_{4}$, which is most in line with the ideal situation. As can be seen from the figure, the points are all distributed near the straight line. Figure 16(b) shows the relationship between the predicted value and the actual value of the model. The closer the point is to the straight line, the smaller the difference between the actual simulation value and the predicted value of the model. According to the above analysis, the prediction models constructed are all reasonable.

Figure 17 shows the three-dimensional response curves of the influence of rotation speed and feed speed on milling parameters on $T_{4}$. It can be clearly seen from Figure 17(a) that, within the range of study parameters, the milling temperature will increase with the increase of rotating speed and decrease with the increase of feeding speed. From the above, it can be concluded that the highest region of milling temperature is concentrated in the region with high rotational speed and low feed speed. It can be seen from Figure 17(b) that, within the range of study parameters, the milling temperature increases with the increase of rotating speed and milling depth. Therefore, when the milling temperature is large, both the rotation speed and the milling 


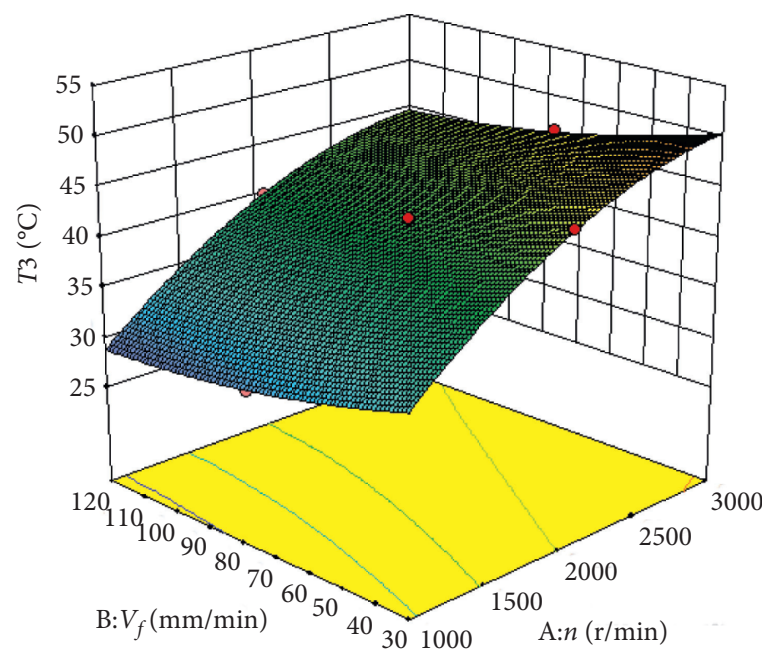

(a)

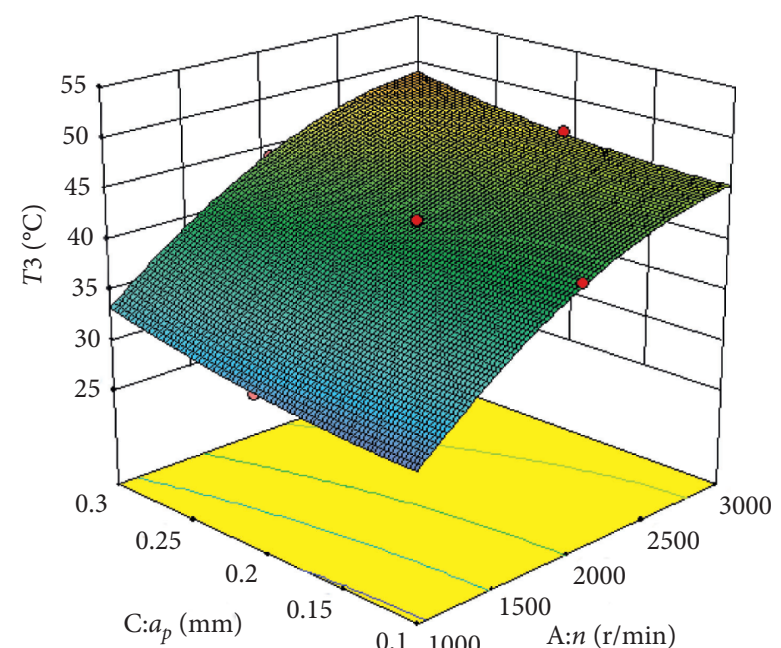

(b)

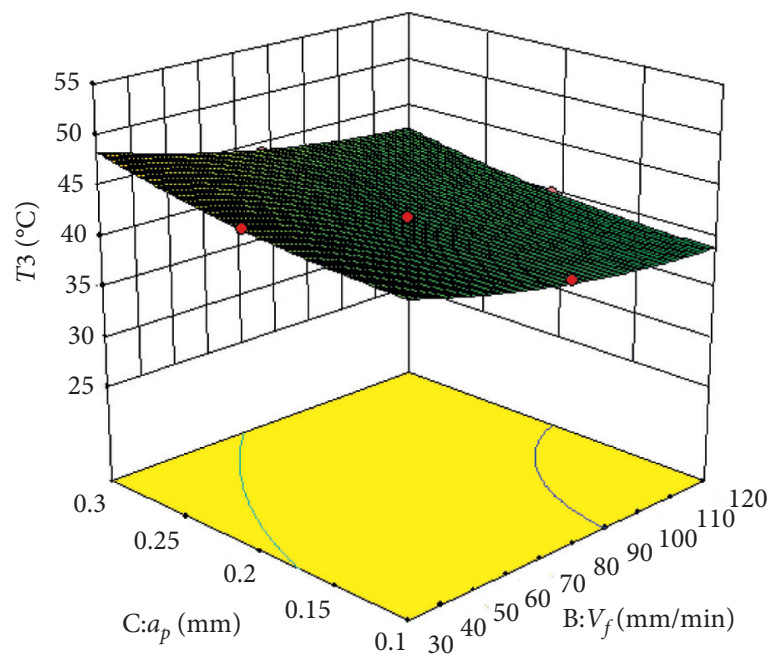

(c)

Figure 15: Response surface curves of the influence of milling parameters on $T_{3}$. (a) Effect of rotation speed $A$ and feed speed $B$ on $T_{3}$, (b) effect of rotation speed $A$ and milling depth $C$ on $T_{3}$, and (c) effect of feed speed $B$ and milling depth $C$ on $T_{3}$.

TABLE 9: Analysis of variance for quadratic regression equation $T_{4}$.

\begin{tabular}{|c|c|c|c|c|c|c|}
\hline Factors & Deviation sum of squares & Degrees of freedom & Mean square error & $F$ & $P($ Prob $>F)$ & \\
\hline Model & 796.83 & 9 & 88.54 & 3370.22 & 0.0341 & Significant \\
\hline Rotation speed $A$ & 643.20 & 1 & 643.20 & 24483.93 & 0.0086 & Significant \\
\hline Feed speed $B$ & 88.80 & 1 & 88.80 & 3380.38 & 0.0243 & Significant \\
\hline Milling depth $C$ & 37.64 & 1 & 37.64 & 1432.64 & 0.0101 & Significant \\
\hline$A B$ & 0.011 & 1 & 0.011 & 0.43 & 0.5276 & \\
\hline$A C$ & 0.031 & 1 & 0.031 & 1.19 & 0.3010 & \\
\hline$B C$ & 0.031 & 1 & 0.031 & 1.19 & 0.3010 & \\
\hline$A^{2}$ & 26.82 & 1 & 26.82 & 1020.78 & 0.0015 & \\
\hline$B^{2}$ & 4.49 & 1 & 4.49 & 170.78 & 0.0002 & \\
\hline$C^{2}$ & 2.12 & 1 & 2.12 & 80.56 & 0.6750 & \\
\hline Residual & 0.26 & 10 & 0.026 & & & \\
\hline Misfit term & 0.094 & 5 & 0.019 & 0.56 & 0.7296 & Not significant \\
\hline Pure error & 0.17 & 5 & 0.034 & & & \\
\hline
\end{tabular}




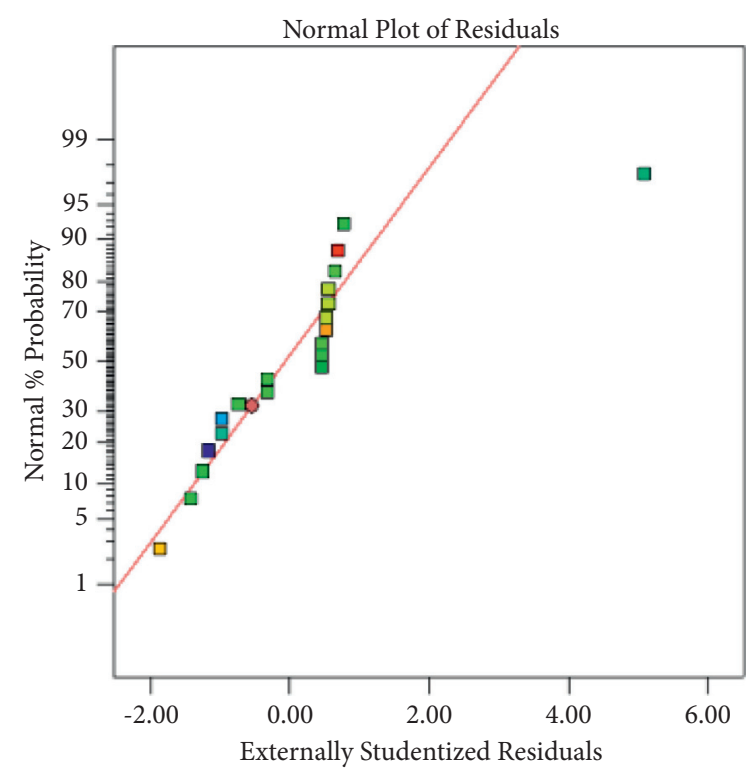

(a)

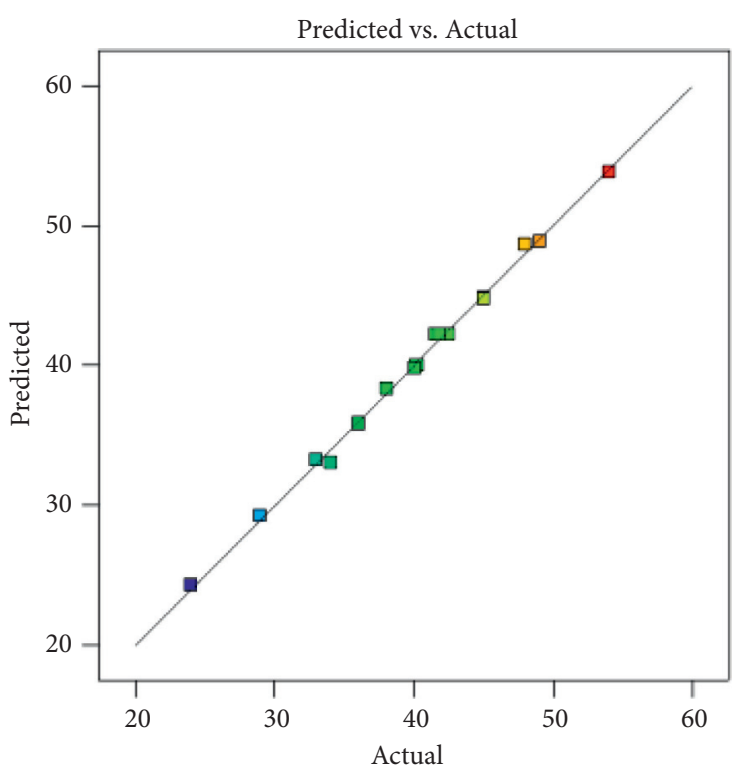

(b)

Figure 16: Residual analysis of prediction equation $T_{4}$. (a) Residual normal probability distribution and (b) relationship between the predicted value and the actual value.

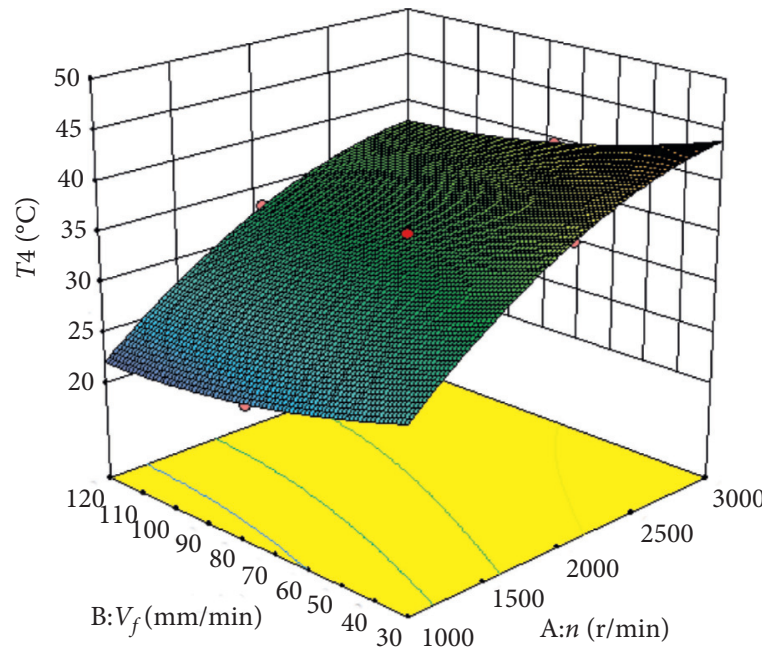

(a)

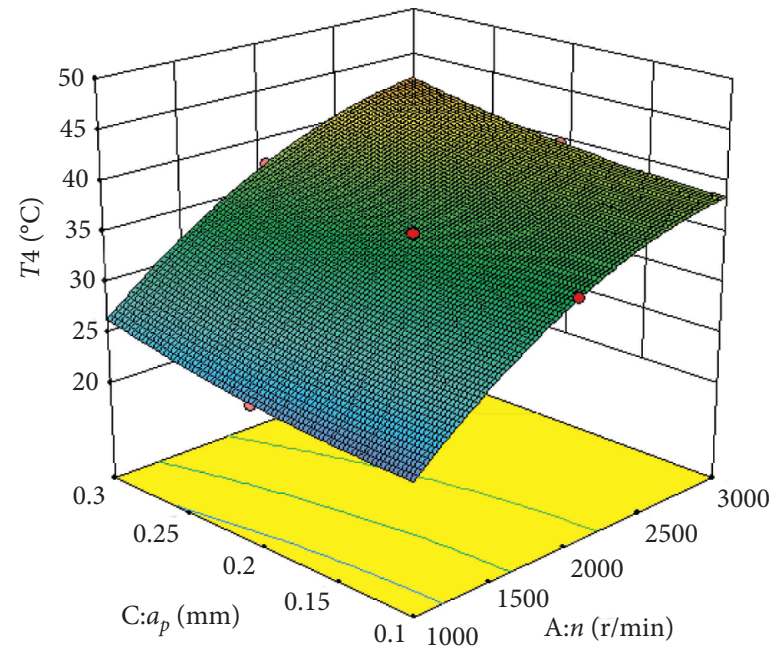

(b)

Figure 17: Continued. 


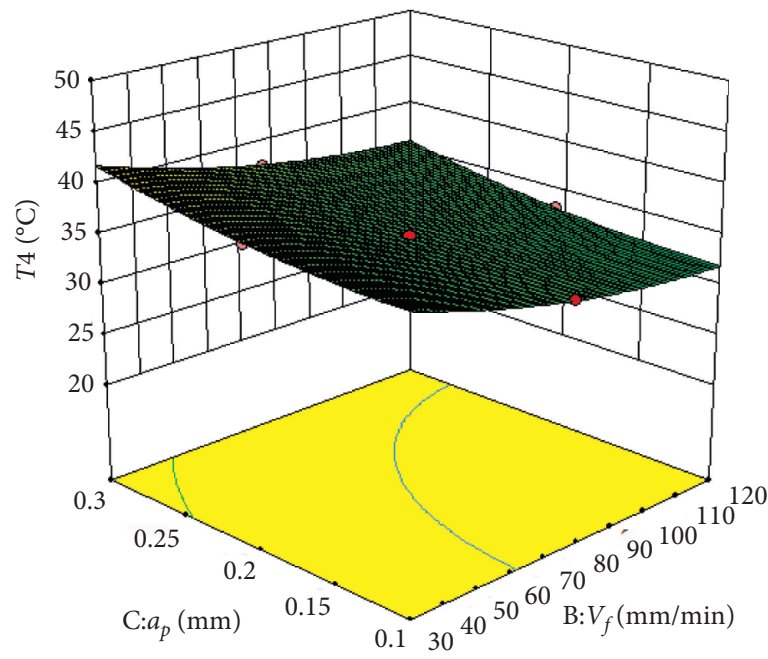

(c)

Figure 17: Response surface curves of the influence of milling parameters on $T_{4}$. (a) Effect of rotation speed $A$ and feed speed $B$ on $T_{4}$, (b) effect of rotation speed $A$ and milling depth $C$ on $T_{4}$, and (c) effect of feed speed $B$ and milling depth $C$ on $T_{4}$.

TABLE 10: First-order sensitivity and global sensitivity of the prediction model.

\begin{tabular}{lccc}
\hline Cutting modes & Cutting parameters & $T$ first-order sensitivity & $T$ global sensitivity \\
\hline & Rotation speed $A$ & 0.66 & 0.68 \\
Parallel cutting mode of cortical bone region & Feed speed $B$ & 0.21 & 0.05 \\
& Milling depth $C$ & 0.68 & 0.23 \\
Cross cutting mode of cortical bone region & Rotation speed $A$ & 0.19 & 0.71 \\
& Feed speed $B$ & 0.05 & 0.22 \\
Vertical cutting mode of cortical bone region & Milling depth $C$ & 0.69 & 0.07 \\
& Rotation speed $A$ & 0.21 & 0.72 \\
Cancellous bone area & Feed speed $B$ & 0.04 & 0.23 \\
& Milling depth $C$ & 0.79 & 0.05 \\
& Rotation speed $A$ & 0.11 & 0.84 \\
& Feed speed $B$ & 0.04 & 0.05 \\
\hline
\end{tabular}

depth are large. It can be seen from Figure 17(c) that, within the range of study parameters, the milling temperature decreases with the increase of feed speed and increases with the increase of milling depth. Therefore, the larger area of milling temperature is concentrated when the feed speed is smaller and the milling depth is larger.

According to the prediction model of the above cortical bone regions under different cutting modes, the anisotropy of bone material has a significant influence on the milling force and milling temperature, which cannot be ignored. Considering the influence of bone units in cortical bone structure, three cutting directions such as parallel to the bone unit, cross with the bone unit, and perpendicular to the bone unit should be used in the cortical bone area. Under the vertical cutting mode, the blade needs to accumulate more energy through the bone unit due to the enhancement of bone unit fiber compared to the other two cutting directions, so the milling temperature is higher than the other two cutting directions. Under the cross cutting mode, the milling crack grows along the bonding line, the matrix, and then the bone unit "peeling." The milling force required for the bone material crack growth or fracture is larger than that in the parallel mode, and the heat generated is correspondingly less. The failure of bone tissue in the parallel cutting mode occurs mainly along the weak bonding line, so the milling temperature is minimal under the parallel cutting mode.

3.3. Global Sensitivity Analysis. The relative contribution rate of each parameter to the output parameter can be obtained by taking the model of milling force and milling temperature in different areas obtained by the response surface method as the objective function and calculating the global sensitivity $S_{T i}$ of the milling parameters of each variable. The first-order sensitivity index and global sensitivity index of each input milling parameter can be obtained, and the influence degree of input milling parameters on output milling force and milling temperature can be obtained by sorting them. The first-order sensitivity and global sensitivity of the prediction model obtained by sensitivity analysis are shown in Table 10 . In order to more intuitively represent the relative 


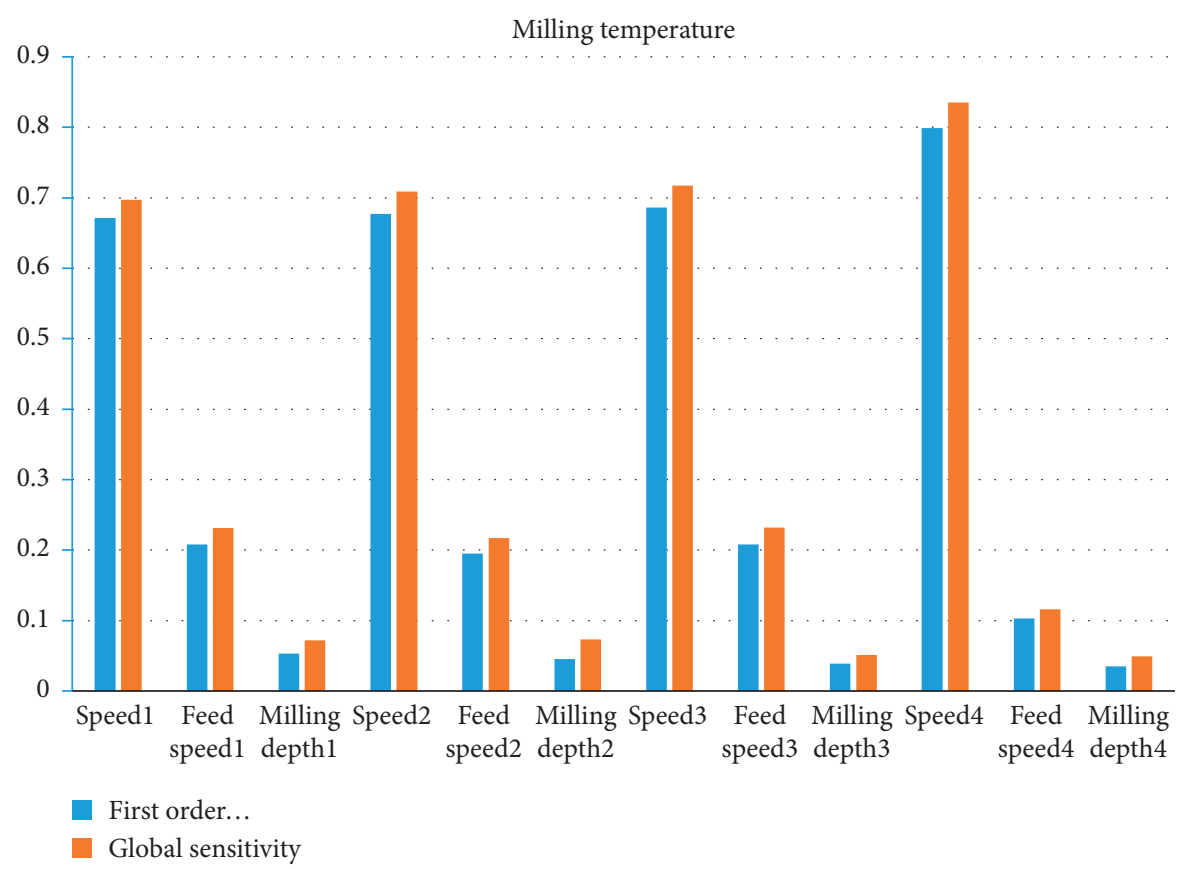

FIGURE 18: First-order sensitivity and global sensitivity of the milling temperature prediction model in different regions.

contributions of different milling parameters in different areas in the above table about milling force and milling temperature, the first-order sensitivity and global sensitivity of the prediction model are expressed in Figure 12.

As can be seen from Figure 18, for a given parameter distribution, the global sensitivity of the milling temperature prediction model is not much different from that of the firstorder sensitivity numerically, so the interaction between the parameters of the respective variables is not particularly obvious. This also verifies the results obtained from the analysis of variance for the prediction model above. It can also be seen that, for the milling temperature model established according to the experiment, the importance and influence of each input parameter on the milling temperature are basically similar under the consideration of different regions and different milling modes. The parameter that has the greatest influence on the milling temperature is the rotation speed, followed by the feed speed, and the influence of the milling depth is not obvious.

\section{Conclusions and Limitations}

(1) Considering the influence of bone microstructure and milling trajectory, the prediction model considering the doctor's surgical skills of the maximum milling temperature under parallel cutting mode, cross cutting mode, and vertical cutting mode in the cortical bone region and the prediction model of milling temperature in cancellous bone regions are established.

(2) By observing the influence curve of milling parameters on milling temperature using the ball milling cutter, it can be seen that, under the same cutting parameters, the milling temperature under the vertical cutting mode is the largest, under the cross cutting mode is the second, and under the parallel cutting mode is the smallest.

(3) It can be seen from the influence curve of milling parameters on milling temperature in cancellous bone region that the highest area of milling temperature is concentrated in the area with high rotating speed and low feeding speed, while the area with large milling temperature is concentrated in the area with large rotating speed and milling depth, small feeding speed, and large milling depth.

(4) By analyzing the parameter sensitivity of the temperature prediction model and the influence of the input parameters on the output milling temperature, it is concluded that rotation speed has the greatest influence on milling temperature, followed by feed speed and with the least influence for milling depth.

(5) In this experiment, doctors operated the spinal surgery cooperative robot for lamina milling, and the fresh pig femur was used instead of human bone, and the thermocouple was used to measure the milling temperature. Although multiple measurements were used to reduce the measurement error, there is still a certain gap between the measurement method and the actual milling temperature. In the future research, we will use human bone as the experiment sample, increase the sample size, and use the infrared thermal imager for temperature measurement, so as to improve the accuracy of the research.

\section{Data Availability}

All data included in this study are available upon request by contact with the corresponding author. 


\section{Conflicts of Interest}

The authors declare no potential conflicts of interest with respect to the research, authorship, and/or publication of this article.

\section{Acknowledgments}

This study was supported by the Natural Science Foundation of Shandong Province (grant no. ZR2020MF099), China Postdoctoral Science Foundation (grant no. 2016M602164), and Qingdao Postdoctoral Researchers Applied Research Foundation (grant no. 2016119).

\section{References}

[1] T. Ginoya, Y. Maddahi, and K. Zareinia, "A historical review of medical robotic platforms," Journal of Robotics, vol. 2021, Article ID 6640031, 13 pages, 2021.

[2] K. Denis, G. Van Ham, J. Vander Sloten et al., "Influence of bone milling parameters on the temperature rise, milling forces and surface flatness in view of robot-assisted total knee arthroplasty," International Congress Series, vol. 1230, no. 1, pp. 300-306, 2001.

[3] D. K. Baro and A. B. Deoghare, "Investigation of cutting force and temperature variation in orthogonal cutting of cortical bone," Materials Today: Proceedings, vol. 5, no. 5, pp. 12595-12608, 2018.

[4] F. Watanabe, Y. Tawada, S. Komatsu, and Y. Hata, "Heat distribution in bone during preparation of implant sites: heat analysis by real-time thermography," The International Journal of Oral \& Maxillofacial Implants, vol. 7, no. 2, pp. 212-219, 1992.

[5] P. A. Federspil, B. Plinkert, and P. K. Plinkert, "Experimental robotic milling in skull-base surgery," Computer Aided Surgery, vol. 8, no. 1, pp. 42-48, 2003.

[6] L. Zhang, L. T. Bruce, W. Guangjun, Z. Kuibang, S. Stephen, and J. S. Albert, "Thermal model to investigate the temperature in bone grinding for skull base neurosurgery," Medical Engineering \& Physics, vol. 35, no. 10, pp. 1391-1398, 2013.

[7] K. I. Al-Abdullah, C. P. Lim, Z. Najdovski, and W. Yassin, "A model-based bone milling state identification method via force sensing for a robotic surgical system," International Journal of Medical Robotics and Computer Assisted Surgery, vol. 15, no. 3, 2019.

[8] B. Kianmajd, D. Carter, and M. Soshi, "A novel toolpath force prediction algorithm using CAM volumetric data for optimizing robotic arthroplasty," International Journal of Computer Assisted Radiology \& Surgery, vol. 11, no. 10, pp. 1-10, 2016.

[9] H. C. Shin and Y. S. Yoon, "Bone temperature estimation during orthopaedic round bur milling operations," Journal of Biomechanics, vol. 39, no. 1, pp. 33-39, 2004.

[10] N. Sugita, T. Osa, and M. Mitsuishi, "Analysis and estimation of cutting-temperature distribution during end milling in relation to orthopedic surgery," Medical Engineering \& Physics, vol. 31, no. 1, pp. 101-107, 2008.

[11] L. T. Bruce, Z. Lihui, W. Anthony, S. Stephen, and J. S. Albert, "Neurosurgical bone grinding temperature monitoring," Procedia CIRP, vol. 5, pp. 226-230, 2013.

[12] K. I. A.-L. Al-Abdullah, H. Abdi, C. P. Lim, and W. A. Yassin, "Force and temperature modelling of bone milling using artificial neural networks," Measurement, vol. 116, pp. 25-37, 2018.
[13] N. Reznikov, R. Shahar, and S. Weiner, "Bone hierarchical structure in three dimensions," Acta Biomaterialia, vol. 10, no. 9, pp. 3815-3826, 2014.

[14] H. Tian, C. Zheng, C. Wang, J. Li, and Y. Yao, "Modeling and simulation of a single abrasive grain micro-grinding force and temperature of bone," Journal of Biomechanical Science and Engineering, vol. 15, no. 4, 2020.

[15] R. Rosolem, H. V. Gupta, W. J. Shuttleworth, X. Zeng, and L. G. G. de Gonçalves, "A fully multiple-criteria implementation of the Sobol' method for parameter sensitivity analysis," Journal of Geophysical Research Atmospheres, vol. 117, 2012. 